\title{
ON THE QUADRATIC SUBFIELD OF A $Z_{2}$-EXTENSION OF AN IMAGINARY QUADRATIC NUMBER FIELD
}

\author{
AKIRA ENDÔ
}

(Communicated by William C. Waterhouse)

\begin{abstract}
We determine explicitly the quadratic subfield of a noncyclotomic $Z_{2}$-extension of an imaginary quadratic number field and get a congruence property of the integer solution of a certain indeterminate equation.
\end{abstract}

1. Introduction. Let $F$ be an imaginary quadratic number field and $Z_{2}$ the additive group of 2-adic rational integers. An infinite normal extension of $F$ with Galois group isomorphic to $Z_{2}$ is called a $Z_{2}$-extension of $F$. It is known that $F$ has two independent $Z_{2}$-extensions [8]. One is $F \subset F(\sqrt{2}) \subset F(\sqrt{2+\sqrt{2}}) \subset \cdots$, which is called a cyclotomic $Z_{2}$-extension. Carrol and Kisilevsky $[\mathbf{3}, 4]$ have shown that the quadratic subfield of a noncyclotomic $Z_{2}$-extension of $F$ is related closely to the 2-primary subgroup $C_{F}(2)$ of the ideal class group of $F$. On the other hand, the structure of $C_{F}(2)$ has already been investigated in detail by Hasse [5-7], Bauer $[1,2]$ and others.

Let $Q$ be the field of rational numbers. In this note we shall treat the case where $F=Q(\sqrt{-p}), p$ an odd prime number, for which $C_{F}(2)$ is cyclic. We shall determine explicitly the quadratic subfield of a noncyclotomic $Z_{2}$-extension of $F$ (Theorems 1 and 3), and show that this problem is also related to a congruence property modulo 8 of the integer solution of a certain indeterminate equation (Theorem 2).

2. Preliminaries. Let $F=Q(\sqrt{-p})$ be as in the Introduction. As is well known, $C_{F}(2) \neq 1$ if and only if $p \equiv 1(\bmod 4)$, and $\left|C_{F}(2)\right| \geq 2^{2}$ if and only if $p \equiv 1(\bmod 8)[5]$. The following was shown or can be easily proved by $[3,4]$ :

PROPOSITION. If $p \equiv 5(\bmod 8)$, then $F(\sqrt{-1}) \subset F(\sqrt{2 E}) \subset F(\sqrt[4]{2 E})$ are subfields of a $Z_{2}$-extension of $F$, where $E$ is ihe fundamental unit of $Q(\sqrt{p})$; if $p \equiv 3$ (mod) and $p=a^{2}+2 b^{2}$, then $F(\sqrt{-2}) \subset F(\sqrt{2 b-a \sqrt{-2}})$ are subfields of $a$ $Z_{2}$-extension of $F$; and if $p \equiv 7(\bmod 8)$ and $p=-a^{2}+2 b^{2}$, then $F(\sqrt{a-\sqrt{-p}})$ is a subfield of a $Z_{2}$-extension of $F$.

In what follows, we assume that $p \equiv 1(\bmod 8)$ and $p=-a^{2}+2 b^{2}$ with $a \equiv$ $1(\bmod 4)$ and $b>0$. (Since $\left(\frac{2}{p}\right)=1$ and $Q(\sqrt{2})$ has a unit with negative norm, such $a, b$ exist.) Then, (2) is ramified in $F:(2)=\mathfrak{z}^{2}$. There exists an ideal $\mathfrak{b}_{1}$ of $F$ such that $\mathfrak{z}_{1}^{2}=(a-\sqrt{-p})$ ad $N \mathfrak{b}_{1}=b$, where $N$ means the norm [5]. The class of $\mathfrak{b}_{1}$ is in $C_{F}^{2}$, where $C_{F}$ is the ideal class group of $F$, if and only if $b \equiv 1(\bmod 4)$.

Received by the editors August 5, 1985 .

1980 Mathematics Subject Classification (1985 Revision). Primary 12A25.

Key words and phrases. Imaginary quadratic number field, $Z_{2}$-extension. 
Now, let $L$ be the maximal abelian 2-ramified (i.e., unramified at all primes different from $z$ ) 2-extension of $F$ with Galois group $G$. Then $L$ contains the composite $K$ of all $Z_{2}$-extensions of $F$ and $G \cong Z_{2} \times Z_{2} \times T$, where $T$ is the torsion subgroup of $G$ corresponding to $K$. Let $A$ denote the subgroup of all elements $\alpha$ of $F^{*}=F-\{0\}$ which are divisible by each prime different from $\mathfrak{z}$ to an even power. Then, in our case, $A$ is generated by $-1,2, a-\sqrt{-p}$, and $F^{* 2}$ [3]. For any $\alpha \in A$, $F(\sqrt{\alpha})$ is 2-ramified, i.e., $F(\sqrt{\alpha}) \subset L$, and further $F(\sqrt{\alpha})$ is contained in $K$ if and only if it is fixed by $T$.

Let $U$ and $J$ be the unit group and the idele group of $F$, respectively. For a prime $\mathfrak{p}$ of $F$, let $U_{\mathfrak{p}}$ denote the unit group of the completion $F_{\mathfrak{p}}$ of $F$ of $\mathfrak{p}$. Put $J^{(2)}=\{1\}_{\mathfrak{z}} \times \prod_{\mathfrak{p \neq z}} U_{\mathfrak{p}}$, a subgroup of $J$. For an abelian group $X$, let $X(2)$ denote the 2-primary torsion subgroup of $X$. By class field theory, $T$ is identified with $\left(J / J^{(2)} F^{*}\right)(2)$. Then the canonical mapping $J / F^{*} \rightarrow C_{F}$ induces an exact sequence

$$
1 \rightarrow H \rightarrow T \stackrel{\phi}{\rightarrow} C_{F}(2),
$$

where $H=\left(U_{z} / U\right)(2) . T / T^{2}$ is considered as a subgroup of $G / G^{2}$ and $G / G^{2}$ is identified with $J / J^{(2)} F^{*} J^{2} . F(\sqrt{\alpha}), \alpha \in A$, is contained in $K$ if and only if

$$
\left(\alpha,\left(x_{\mathfrak{p}}\right)\right)=\prod_{\mathfrak{p}}\left(\alpha, x_{\mathfrak{p}}\right)_{\mathfrak{p}}=1
$$

for all $\left(x_{\mathfrak{p}}\right) \in T=\left(J / J^{(2)} F^{*}\right)(2)$, where $(,)_{\mathfrak{p}}$ is the Hilbert 2-symbol in $F_{\mathfrak{p}}$. Carroll [3] calculated $\left(\alpha,\left(x_{\mathfrak{p}}\right)\right)$ for $\alpha \in A,\left(x_{\mathfrak{p}}\right) \in T$ in the case where $\left|T^{2}\right| \leq 2$.

Now, denote by

$$
\left(\ldots, \underset{\mathfrak{p}_{i}}{\mathfrak{p}_{i}}, \ldots\right)
$$

an idele of $F$ having $x_{\mathfrak{p}_{i}}$ at $\mathfrak{p}_{i}$-components and 1 elsewhere. In our case $T$ is cyclic and $H$ is its subgroup of order $2[3]$. We here note that $F_{z}=Q_{2}(\sqrt{-1}), Q_{2}$ being the field of 2 -adic rational integers. It is seen that $H$ is generated by

$$
(\sqrt{-1}, \ldots) \text {. }
$$

Let

$$
z_{0}=(1-\sqrt{-1}, \ldots) \in J
$$

Then

$$
z_{0}^{2} \equiv(\sqrt{-1}, \ldots) \quad\left(\bmod J^{(2)} F^{*}\right)
$$

3. Theorems. As stated above, $A / F^{* 2}$ is generated by $-1,2$, and $a-\sqrt{-p}$. $F(\sqrt{2})$ is contained in the cyclotomic $Z_{2}$-extension of $F$. We assume that $\sqrt{-p} \equiv$ $\sqrt{-1}\left(\bmod \mathfrak{z}^{4}\right)$ and so $\sqrt{p} \equiv 1(\bmod 4)$ in $Q_{2}$. We now calculate $\left(-1, z_{0}\right)$ and $\left(a-\sqrt{-p}, z_{0}\right)$. For a rational prime $q,(,)_{q}$ denotes the Hilbert 2-symbol at the field of $q$-adic rational numbers.

$$
\begin{aligned}
\left(-1, z_{0}\right) & =(-1,1-\sqrt{-1})_{\mathfrak{z}}=(\sqrt{-1}, 1-\sqrt{-1})_{\mathfrak{z}}^{2}=1, \\
\left(a-\sqrt{-p}, z_{0}\right) & =(a-\sqrt{-p}, 1-\sqrt{-1})_{\mathfrak{z}}=(\sqrt{p}(1-\sqrt{-1}), 1-\sqrt{-1})_{\mathfrak{z}} \\
& =(\sqrt{p}, 1-\sqrt{-1})_{\mathfrak{z}}=(\sqrt{p}, 2)_{2}=(-1)^{(p-1) / 8} .
\end{aligned}
$$


Thus, if $p \equiv 9(\bmod 16)$, then $F(\sqrt{-1}) \subset K, T=\left\langle z_{0}\right\rangle$ and $\phi(T) \neq C_{F}(2)$. If $p \equiv$ $1(\bmod 16)$, then it remains to determine whether $F(\sqrt{-1}) \subset K$ or $F(\sqrt{ \pm a-\sqrt{-p}})$ $\subset K$.

From now on we assume $p \equiv 1(\bmod 16) ;$ then $\sqrt{p} \equiv 1(\bmod 8)$. Each prime $q_{1} \mid b$ splits in $F:\left(q_{1}\right)=\mathfrak{q}_{1} \overline{q_{1}}$. We may assume that $\mathfrak{q}_{1} \mid(a-\sqrt{-p})$ and hence $\mathfrak{q}_{1} \mid \mathfrak{b}_{1}$. Let $\mu_{1}=(a-\sqrt{-p}) /(1-\sqrt{-1}) \in F_{z}=Q_{2}(\sqrt{-1}) ;$ then $\mu_{1} \equiv 1\left(\bmod z^{5}\right)$ and hence $\sqrt{\mu_{1}} \equiv 1\left(\bmod \mathfrak{z}^{3}\right)$ exists in $F_{\mathfrak{z}}$. Define

$$
z_{1}=\left(\underset{\mathfrak{z}}{\sqrt{\mu_{1}}}, \ldots, \underset{\mathfrak{q}_{1} \mid \mathfrak{b}_{1}}{b_{1}}, \ldots\right) \in J
$$

where $b_{1}=b . \phi\left(z_{1}\right)$ is the class of $\mathfrak{b}_{1}$ and

$$
\begin{aligned}
& \left.z_{1}^{2}=\underset{\mathfrak{z}}{\left(\mu_{1}, \ldots, \mathfrak{q}_{1} \mid \mathfrak{b}_{1}\right.} b_{1}^{2}, \ldots\right) \\
& \equiv \frac{1}{a-\sqrt{-p}}\left(\mu_{\mathfrak{z}}, \ldots, \underset{\mathfrak{q}_{1} \mid \mathfrak{b}_{1}}{b_{1}^{2}}, \ldots\right) \equiv z_{0}^{-1} \quad\left(\bmod J^{(2)} F^{*}\right) .
\end{aligned}
$$

It is easy to see that

$$
\begin{aligned}
\sqrt{\mu_{1}} & \equiv 1+\frac{a-\sqrt{p}}{4}(1-\sqrt{-1})+\frac{\sqrt{p}-1}{2} \quad\left(\bmod \mathfrak{z}^{5}\right) \\
& \equiv \begin{cases}1,-1-2 \sqrt{-1}\left(\bmod \mathfrak{z}^{5}\right), & a \equiv 1(\bmod 16) \\
5,-1+2 \sqrt{-1}\left(\bmod \mathfrak{z}^{5}\right), & a \equiv 9(\bmod 16)\end{cases}
\end{aligned}
$$

Then,

$$
\begin{aligned}
\left(-1, z_{1}\right) & =\left(-1, \sqrt{\mu_{1}}\right)_{\mathfrak{z}} \prod_{\mathfrak{q}_{1} \mid \mathfrak{b}_{1}}(-1, b)_{\mathfrak{q}_{1}} \\
& =\prod_{q_{1} \mid b}(-1, b)_{q_{1}}=(-1, b)_{2}=(-1)^{(b-1) / 2} \\
\left(a-\sqrt{-p}, z_{1}\right) & =\left(a-\sqrt{-p}, \sqrt{\mu_{1}}\right)_{\mathfrak{z}} \prod_{\mathfrak{q}_{1} \mid \mathfrak{b}_{1}}(a-\sqrt{-p}, b)_{\mathfrak{q}_{1}} \\
& =\left(1-\sqrt{-1}, \sqrt{\mu_{1}}\right)_{\mathfrak{z}} \prod_{\mathfrak{q}_{1} \mid \mathfrak{b}_{1}}(2(a+\sqrt{-p}), b)_{\mathfrak{q}_{1}} \\
& =(-1)^{(a-1) / 8} \prod_{\mathfrak{q}_{1} \mid \mathfrak{b}_{1}}(4 a, b)_{\mathfrak{q}_{1}} \\
& =(-1)^{(a-1) / 8} \prod_{q_{1} \mid b}(a, b)_{q_{1}}=(-1)^{(a-1) / 8}\left(\frac{a}{b}\right) .
\end{aligned}
$$

Herein we have used that $(1-\sqrt{-1}, 5)_{\mathfrak{z}}=(2,5)_{2}=-1,(1-\sqrt{-1},-1-2 \sqrt{-1})_{\mathfrak{z}}$ $=\left(1-\sqrt{-1}, 1-(1+\sqrt{-1})^{2}(1-\sqrt{-1})\right)_{\mathfrak{z}}=1$ and $(1-\sqrt{-1},-1+2 \sqrt{-1})_{\mathfrak{z}}=$ $(1-\sqrt{-1}, 5(-1-2 \sqrt{-1}))_{z}=-1$. Hence we have the following theorem.

THEOREM 1. Suppose that $p \equiv 1(\bmod 16)$ and $p=-a^{2}+2 b^{2}$ with $a \equiv$ $1(\bmod 8)$ and $b>0$. If $b \equiv 1(\bmod 4)$ and $\left(\frac{a}{b}\right)=-(-1)^{(a-1) / 8}$, then $F(\sqrt{-1})$ is $a$ subfield of $a Z_{2}$-extension of $F$ and $\phi(T) \neq C_{F}(2) ;$ and if $b \equiv-1(\bmod 4)$, then $F(\sqrt{a-\sqrt{-p}})$ or $F(\sqrt{-a-\sqrt{-p}})$ is a subfield of a $Z_{2}$-extension of $F$ according as $\left(\frac{a}{b}\right)=(-1)^{(a-1) / 8}$ or $(-1)^{(a-1) / 8}$, and $\phi(T)=C_{F}(2)$. In these cases, $T=\left\langle z_{1}\right\rangle$. 
Let $b^{\prime}$ be the square-free part of $b$ and put $b=b^{\prime} g^{2}$ with $g>0$. If $b \equiv b^{\prime} \equiv$ $1(\bmod 4)$, then there exist rational integers $r, s, t$ which satisfy

$$
r^{2}+p s^{2}-b^{\prime} t^{2}=0, \quad(r, s, t)=1, t>0 .
$$

Let $c=\prod_{l}^{\prime} l^{v_{l}\left(b^{\prime}\right)}$ and $d=\prod_{l}^{\prime \prime} l^{v_{l}(g, t)}$, where the ${ }^{\prime}$ (resp. ") indicates that $l$ runs through rational primes dividing $(a-\sqrt{-1})(r-s \sqrt{-p})(\operatorname{resp} .(a-\sqrt{-p})(r+s \sqrt{-p}))$ and $l^{v_{l}(x)}$ means the exact power of $l$ dividing $x$. Bauer [1] showed that there exists a primitive ideal $\mathfrak{b}_{2}$ of $F$ such that

$$
\mathfrak{b}_{1}{\overline{\mathfrak{b}_{2}}}^{2}=\left(\left(c g^{2} / d^{2}\right)(r-s \sqrt{-p})\right), \quad N \mathfrak{b}_{2}=c g t / d^{2} .
$$

Put $b_{2}=c g t / d^{2}$. The class of $\mathfrak{b}_{2}$ is in $C_{F}^{2}$ if and only if $b_{2} \equiv 1(\bmod 4)$. We here note that $c=\left(r+a s, b^{\prime}\right)$ and $d=(r-a s, g, t)$.

Each prime $q_{2} \mid t$ splits in $F:\left(q_{2}\right)=\mathfrak{q}_{2} \overline{q_{2}}$. We assume that $\mathfrak{q}_{2} \mid r+s \sqrt{-p}$ and hence $\mathfrak{q}_{2} \mid \overline{\mathfrak{b}_{1}} \mathfrak{b}_{2}$. Note that for a prime $q_{1} \mid b$, if $q_{1} \mid b_{2}$ and $q_{1} \nmid t$, then $\mathfrak{q}_{1} \mid \mathfrak{b}_{2}$. It is easy to see that $r$ and $s$ are of mixed parity (i.e., one odd and one even) and that $4 \mid r s$ if and only if $p \equiv 2 a-1(\bmod 32)$.

THEOREM 2. Suppose that $p=-a^{2}+2 b^{2} \equiv 1(\bmod 16), a \equiv 1(\bmod 8), b>0$ and $b \equiv 1(\bmod 4)$, and let $b^{\prime}$ be the square-free part of $b$. If rational integers $r, s, t$ satisfy $r^{2}+p s^{2}=b^{\prime} t^{2},(r, s, t)=1, t>0$, then

$$
r+s \equiv \begin{cases} \pm c+4 \varsigma(\bmod 8), & \left(\frac{a}{b}\right)=(-1)^{(a-1) / 8} \\ \pm 5 c+4 \varsigma(\bmod 8), & \left(\frac{a}{b}\right)=(-1)^{(a-1) / 8+1}\end{cases}
$$

where $c=\left(r+a s, b^{\prime}\right)$ and $\varsigma=0$ or 1 such that

$$
\varsigma \equiv \begin{cases}\frac{1}{16}(p-1)(\bmod 2), & s \equiv 0(\bmod 4) \text { or } r \equiv 0(\bmod 2), \\ \frac{1}{16}(p-1)+1(\bmod 2), & s \equiv 2(\bmod 4) .\end{cases}
$$

Proof. As shown above, if $b \equiv 1(\bmod 4)$, then $z_{1} \in T^{2}$ or not, in other words, $z_{1}$ is the square of some $z_{2} \in J$ modulo $J^{(2)} F^{*}$ or not, according as $\left(\frac{a}{b}\right)=(-1)^{(a-1) / 8}$ or $(-1)^{(a-1) / 8+1}$. We may assume that $\phi\left(z_{2}\right)$ is the class of $\mathfrak{b}_{2}$. Therefore such $z_{2}$ is of the form

$$
z_{2}=\left(\underset{\mathfrak{z}}{\sqrt{\mu_{2}}}, \ldots, \underset{\mathfrak{q}_{1} \mid \mathfrak{b}_{1}, \mathfrak{b}_{2}}{b_{2}}, \ldots, \underset{\substack{\mathfrak{q}_{2} \nmid \mathfrak{b}_{1} \\ \mathfrak{q}_{2} \mid \mathfrak{b}_{2}}}{b_{2}}, \ldots\right) .
$$

Since

$$
(2, \ldots) \equiv 1 \quad\left(\bmod J^{(2)} F^{*}\right)
$$

we may assume that $\mu_{2}$ is prime to $\mathfrak{z}$. There exists $M \in F$ such that

$$
M\left(\underset{\mathfrak{z}}{\mu_{2}}, \ldots, \underset{\mathfrak{q}_{1} \mid \mathfrak{b}_{1}, \mathfrak{b}_{2}}{b_{2}^{2}}, \ldots, \underset{\substack{\mathfrak{q}_{2} \nmid \mathfrak{b}_{1} \\ \mathfrak{q}_{2} \mid \mathfrak{b}_{2}}}{b_{2}^{2}}, \ldots\right) \equiv\left(\sqrt{\mu_{1}}, \ldots, \underset{\mathfrak{q}_{1} \mid \mathfrak{b}_{1}}{b_{1}}, \ldots\right)\left(\bmod J^{(2)}\right) .
$$

Then $M \mu_{2}=\sqrt{\mu_{1}}$ and $(M) \mathfrak{b}_{2}^{2}=\mathfrak{b}_{1}$, because $N \mathfrak{b}_{1}=b_{1}$ and $N \mathfrak{b}_{1}=b_{2}$. Since $\mathfrak{b}_{1}^{-1} \mathfrak{b}_{2}^{2}=\left(\left(b_{2} / b^{\prime} g t\right)(r+s \sqrt{-p})\right), 1 / M=\mu_{2} / \sqrt{\mu_{1}}= \pm\left(b_{2} / b^{\prime} g t\right)(r+s \sqrt{-p})$. Thus we have that, since

$$
z_{0}^{2} \equiv(\sqrt{-1}, \ldots)(\in H) \quad\left(\bmod J^{(2)} F^{*}\right)
$$


$\sqrt{-1}^{j} \mu_{2}= \pm \sqrt{-1}^{j}\left(b_{2} / b^{\prime} g t\right)(r+s \sqrt{-p}) \sqrt{\mu_{1}}, j=0$ or 1 , is a square in $Q_{2}(\sqrt{-1})$, i.e., $\equiv \pm 1\left(\bmod \mathfrak{z}^{5}\right)$, if and only if $\left(\frac{a}{b}\right)=(-1)^{(a-1) / 8}$. Since

$$
\begin{aligned}
& \mu_{2} \equiv \pm \frac{b_{2}}{b^{\prime} g t}(r+s \sqrt{-1}) \sqrt{\mu_{1}} \equiv \pm \frac{c}{b}(r+s \sqrt{-1}) \sqrt{\mu_{1}} \quad\left(\bmod \mathfrak{z}^{5}\right) \\
& \equiv \begin{cases} \pm c(r+s \sqrt{-1})\left(\bmod \mathfrak{z}^{5}\right), & p \equiv 2 a-1 \equiv 1(\bmod 32), \\
\pm 5 c(r+s \sqrt{-1})\left(\bmod \mathfrak{z}^{5}\right), & p \equiv 2 a-1 \equiv 17(\bmod 32), \\
\pm 5(-1+2 \sqrt{-1}) c(r+s \sqrt{-1})\left(\bmod \mathfrak{z}^{5}\right), & p \equiv 2 a-17 \equiv 1(\bmod 32), \\
\pm 5(-1-2 \sqrt{-1}) c(r+s \sqrt{-1})\left(\bmod \mathfrak{z}^{5}\right), & p \equiv 2 a-17 \equiv 17(\bmod 32),\end{cases}
\end{aligned}
$$

the assertion of the theorem follows easily.

We next consider the case where $b \equiv 1(\bmod 4)$ and $\left(\frac{a}{b}\right)=(-1)^{(a-1) / 8}$. We may assume $r+s \equiv c(\bmod 4)$. Then, from the proof above

$$
\frac{b_{2}}{b^{\prime} g t}(r+s \sqrt{-p}) \sqrt{\mu_{1}} \equiv \begin{cases}1\left(\bmod \mathfrak{z}^{5}\right), & s \equiv 0(\bmod 2), \\ \sqrt{-1}\left(\bmod \mathfrak{z}^{5}\right), & r \equiv 0(\bmod 2) .\end{cases}
$$

We normalize $\mu_{2}$ as follows:

$$
\mu_{2}= \begin{cases}\frac{b_{2}}{b^{\prime} g t}(r+s \sqrt{-p}) \sqrt{\mu_{1}}, & s \equiv 0(\bmod 2), \\ \frac{b_{2}}{b^{\prime} g t}(r+s \sqrt{-p}) \frac{\sqrt{\mu_{1}}}{\sqrt{-1}}, & r \equiv 0(\bmod 2) .\end{cases}
$$

Then $\mu_{2} \equiv 1\left(\bmod \mathfrak{z}^{5}\right)$ and hence $\sqrt{\mu_{2}} \equiv 1\left(\bmod \mathfrak{z}^{3}\right)$ exists in $Q_{2}(\sqrt{-1})$. Put

$$
\begin{aligned}
& z_{2}=\left(\underset{\mathfrak{z}}{\sqrt{\mu_{2}}}, \ldots, \underset{\mathfrak{q}_{1} \mid \mathfrak{b}_{1}, \mathfrak{b}_{2}}{b_{2}}, \ldots, \underset{\mathfrak{q}_{2}+\mathfrak{b}_{1}}{b_{2}}, \ldots\right) \in J \\
& \mathfrak{q}_{2} \mid \mathfrak{b}_{2}
\end{aligned}
$$

then $z_{2}^{2} \equiv z_{1}\left(\bmod J^{(2)} F^{*}\right)$ if $2 \mid s$ and $\left(z_{2} z_{0}^{-1}\right)^{2} \equiv z_{1}\left(\bmod J^{(2)} F^{*}\right)$ if $2 \mid r$. It is easy to see that

$$
\sqrt{\mu_{2}} \equiv 1+2 \gamma(1-\sqrt{-1})+4 \delta \quad\left(\bmod z^{5}\right)
$$

with

$$
\begin{aligned}
& \gamma=\left\{\begin{array}{l}
\frac{1}{16}(a-\sqrt{p}+4 r s), \quad s \equiv 0(\bmod 2), \\
\frac{1}{16}(a-\sqrt{p}-4 r s), \quad r \equiv 0(\bmod 2),
\end{array}\right. \\
& \delta= \begin{cases}\frac{1}{16}\left(s^{2}+2 r s-\left(\frac{b_{2}}{b^{\prime} g t}\right)^{2} r^{2} \sqrt{p}+1\right), & s \equiv 0(\bmod 2), \\
\frac{1}{16}\left(r^{2}-2 r s-\left(\frac{b_{2}}{b^{\prime} g t}\right)^{2} p s^{2} \sqrt{p}+1\right), & r \equiv 0(\bmod 2) .\end{cases}
\end{aligned}
$$

Put $\rho=16(\gamma-\delta)$; then

$$
\rho \equiv \begin{cases}-s^{2}+2 r s+\left(\frac{b_{2}}{b^{\prime} g t}\right)^{2} a r^{2}-1(\bmod 32), & s \equiv 0(\bmod 2), \\ -r^{2}-2 r s+\left(\frac{b_{2}}{b^{\prime} g t}\right)^{2} a p s^{2}-1(\bmod 32), & r \equiv 0(\bmod 2) .\end{cases}
$$


Hence

$$
\begin{aligned}
& \left(-1, z_{2}\right)=\left(-1, \sqrt{\mu_{2}}\right)_{\mathfrak{z}} \prod_{\mathfrak{q}_{1} \mid \mathfrak{b}_{1}, \mathfrak{b}_{2}}\left(-1, b_{2}\right)_{\mathfrak{q}_{1}} \prod_{\substack{\mathfrak{q}_{2}+\mathfrak{b}_{1} \\
\mathfrak{q}_{2} \mid \mathfrak{b}_{2}}}\left(-1, b_{2}\right)_{\mathfrak{q}_{2}} \\
& =\prod_{q_{1} \mid b_{1}, b_{2}}\left(-1, b_{2}\right)_{\mathfrak{q}_{1}} \prod_{\substack{q_{2}+b_{1} \\
q_{2} \mid b_{2}}}\left(-1, b_{2}\right)_{q_{2}} \\
& =\left(-1, b_{2}\right)_{2}=(-1)^{\left(b_{2}-1\right) / 2} \text {, }
\end{aligned}
$$

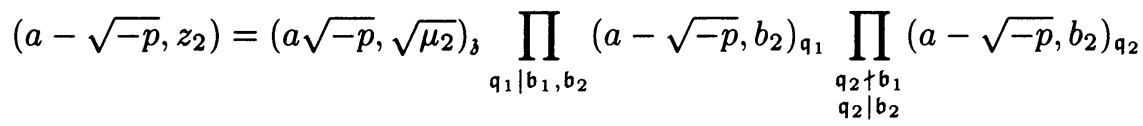

$$
\begin{aligned}
& =\left(1-\sqrt{-1}, \sqrt{\mu_{2}}\right)_{\mathfrak{z}} \prod_{\mathfrak{q}_{1} \mid \mathfrak{b}_{1}, \mathfrak{b}_{2}}\left(2(a+\sqrt{-p}), b_{2}\right)_{\mathfrak{q}_{1}} \prod_{\substack{\mathfrak{q}_{2}+\mathfrak{b}_{1} \\
\mathfrak{q}_{2} \mid \mathfrak{b}_{2}}}\left((r+a s) s, b_{2}\right)_{\mathfrak{q}_{2}} \\
& =(-1)^{\rho / 16} \prod_{q_{1} \mid b_{1}, b_{2}}\left(a, b_{2}\right)_{q_{1}} \prod_{\substack{q_{2}+b_{1} \\
q_{2} \mid b_{2}}}\left((r+a s) s, b_{2}\right)_{q_{2}}=(-1)^{\rho / 16} \chi
\end{aligned}
$$

with

$$
\chi=\prod_{q_{1} \mid b_{1}}\left(\frac{a}{q_{1}}\right)^{v_{q_{1}}\left(b_{2}\right)} \prod_{\substack{q_{2}+b_{1} \\ q_{2} \mid b_{2}}}\left(\frac{(r+a s) s}{q_{2}}\right)^{v_{q_{2}}\left(b_{2}\right)} .
$$

Therefore the following theorem holds:

THEOREM 3. Suppose that $p=-a^{2}+2 b^{2} \equiv 1(\bmod 16), a \equiv 1(\bmod 8), b>$ $0, b \equiv 1(\bmod 4)$ and $\left(\frac{a}{b}\right)=(-1)^{(a-1) / 8}$, and let $b=b^{\prime} g^{2}$ with square-free $b^{\prime}$ and $g>0$. Take rational integers $r, s, t$ such that $r^{2}+p s^{2}=b^{\prime} t^{2},(r, s, t)=1$, $r+s \equiv\left(r+a s, b^{\prime}\right)(\bmod 4), t>0$, and define $b_{2}, \rho, \chi$ as above. If $b_{2} \equiv 1(\bmod 4)$ and $\chi=(-1)^{\rho / 16+1}$, then $F(\sqrt{-1})$ is a subfield of a $Z_{2}$-extension of $F$ and $\phi(T) \neq C_{F}(2)$; and if $b_{2} \equiv-1(\bmod 4)$, then $F(\sqrt{a-\sqrt{-p}})$ or $F(\sqrt{-a-\sqrt{-p}})$ is a subfield of $a$ $Z_{2}$-extension of $F$ according as $\chi=(-1)^{\rho / 16}$ or $(-1)^{\rho / 16+1}$, and $\phi(T)=C_{F}(2)$. In these cases, $T=\left\langle z_{2}\right\rangle$.

In the case where $b_{2} \equiv 1(\bmod 4)$ and $\chi=(-1)^{\rho / 16}, T$ has a proper subgroup $\left\langle z_{2}\right\rangle$ and we can also determine the quadratic subfield of a noncyclotomic $Z_{2}$-extension of $F$ after a finite number of procedures similar to the above, since $C_{F}(2)$ is a finite group.

\section{REFERENCES}

1. H. Bauer, Zur Berechnung der 2-Klassenzahl der quadratischen Zahlkörper mit genau zwei verschiedenen Diskriminantenprimteilern, J. Reine Angew. Math. 248 (1971), 42-46.

2. __ Die 2-Klassenzahlen specieller quadratischer Zahlkörper, J. Reine Angew. Math. 252 (1972), 79-81.

3. J. E. Carroll, On determining the quadratic subfields of $Z_{2}$-extensions of complex quadratic fields, Compositio Math. 30 (1975), 259-271.

4. J. E. Carroll and H. Kisilevsky, Initial layers of $Z_{2}$-extensions of complex quadratic fields, Compositio Math. 3 (1976), 157-168.

5. H. Hasse, Über die Klassenzahl des Körpers $P(\sqrt{-p})$ mit einer Primzahl $p \equiv 1$ mod $2^{3}$, Aequationes Math. 3 (1969), 165-169. 
6. __ Über die Teilbarkeit durch $2^{3}$ der Klassenzahl imaginär-quadratischer Zahlkörper mit genau zwei verschiedenen Diskriminantenprimteilern, J. Reine Angew. Math. 241 (1970), 1-6.

7. __ An algorithm for determining the structure of the 2-Sylow subgroup of the divisor class group of a quadratic number field, Sympos. Math. 15 (1975), 341-352.

8. K. Iwasawa, On $Z_{l}$-extensions of algebraic number fields, Ann. of Math. (2) 98 (1973), 247-326.

Department of Mathematics, Kumamoto University, Kumamoto, Japan 